\title{
Why Integrating Research and Scholarship into Dental Education Matters
}

\author{
Peter J. Polverini, D.D.S., D.M.Sc.
}

$\mathrm{D}$ entistry has embraced research innovation and scholarship as core values. The knowledge generated through research both informs and influences practice by paving the way for evidence-based decision making. Participation in research and scholarship is an important part of a dental student's educational experience. At a minimum, research engages a student's intellectual curiosity, satisfies an inherent thirst for discovery, and provides an outlet for creativity. More importantly, research deepens a student's understanding and appreciation for the profession of dentistry and produces a practitioner who is better able to assess and incorporate new knowledge into practice.

In this era of health care reform, dentistry will migrate from a reactive to a prospective form of health care. As new technologies evolve, dental schools will be expected to provide students with the knowledge and skills needed to assess disease risk, predict disease onset with accuracy and reliability, and intervene early to halt disease progression. It is through research and discovery that dental schools will prepare future practitioners to remain ahead of this emerging health care curve, stay relevant and up-to-date, and be better prepared to meet future challenges during a time of great uncertainty.

Unfortunately, opportunities for dental students to engage in research and scholarship are becoming a luxury for most, rather than a staple of the educational experience. This trend challenges the long-held view that research and scholarship have inherent value in dental education and are integral to shaping the future of dental practice. This challenge has not gone unnoticed by students. In this issue of the journal, the American Association for Dental Research's National Student Research Group reports results of a survey of dental students' attitudes about research and scholarship and the barriers they face in accessing scholarly opportunities. The results of this study raise a number of concerns. Students indicated that a major obstacle to research participation is lack of sufficient time in the curriculum. Despite an increased emphasis on evidence-based practice, these students expressed concern that they are ill prepared to evaluate evidence using disciplines such as biostatistics and research methodology. They reported believing that rapid advances in technology will necessitate greater emphasis on integrating research and scholarship in the curriculum. These students also lamented that, despite a research standard for accreditation, progress in implementing scholarly activity in dental curricula is proceeding at a slow pace. These students noted that it is imperative that dental schools explore innovative strategies to address research infrastructure and the shortage of faculty capable of mentoring students in research and scholarship. Finally, these students expressed concern that they are woefully unprepared and/or lack skills in critical thinking to evaluate and assess the science that underpins decision making in their profession.

With dwindling resources, dental schools must make difficult choices and manage competing interests. Infrastructure costs can be prohibitive for schools that wish to develop new or expand existing research programs. As we migrate toward a more collaborative care environment, there may be opportunities to reduce costs and maximize resources while improving care. A similarly collaborative approach is long overdue in the research community. Research and discovery can be embedded in curricula by forming regional research consortia, developing collaborative training and mentoring programs for faculty members, and transitioning the school's clinical environment into practice-based networks. Alternatively, our current cookie cutter approach to dental education may have to be rethought. Perhaps a greater emphasis on collaborative, interdisciplinary learning may prompt dental schools to take a more progressive approach to health science education and practice. Many stress that experiential learning in community-based settings enhances a student's understanding and appreciation of the environmen- 
tal, psychosocial, and cultural determinants of oral health. Research and scholarship should also be considered an essential experiential learning opportunity.

Because health care will continue to evolve, it is imperative that we prepare our students for this dynamic future. That preparation should include a range of research and scholarly experiences that will equip students with the tools required to evaluate and assess the most current literature and scientific discoveries that will shape the future of dental practice.

Dr. Polverini is Professor of Dentistry and Pathology, University of Michigan School of Dentistry, 1011 North University Avenue, Ann Arbor, MI 48109-1078; neovas@umich.edu. He is President of the American Association for Dental Research. 